\title{
Individual Differences in Nicotine Dependence, Withdrawal Symptoms, and Sex Predict Transient fMRI-BOLD Responses to Smoking Cues
}

\author{
Francis J McClernon*,', Rachel V Kozink' and Jed E Rose' \\ 'Department of Psychiatry and Behavioral Sciences, Duke University Medical Center, Durham, NC, USA
}

\begin{abstract}
Exposure to smoking cues increases craving for cigarettes and can precipitate relapse. Whereas brain imaging studies have identified a distinct network of brain regions subserving the processing of smoking cues, little is known about the influence of individual difference factors and withdrawal symptoms on brain cue reactivity. Multiple regression analysis was used to evaluate relations between individual difference factors and withdrawal symptoms and event-related blood oxygen level-dependent responses to visual smoking cues in a sample of 30 smokers. Predictors were self-report nicotine dependence (Fagerström test of nicotine dependence, FTND), prescan withdrawal symptoms (craving and negative affect), and sex. The unique variance of each predictor was examined after controlling for each of the others. Positive associations were observed between FTND and reactivity to cues in right anterior cingulate and orbitofrontal cortex (OFC) whereas negative associations were observed between prescan craving and reactivity in ventral striatum. Higher negative affect or being male was associated with greater reactivity in left hippocampus and left OFC. Women exhibited greater cue reactivity than men in regions including the cuneus and left superior temporal gyrus. Individual difference factors and withdrawal symptoms were uniquely associated with brain reactivity to smoking cues in regions subserving reward, affect, attention, motivation, and memory. These findings provide further evidence that reactivity to conditioned drug cues is multiply determined and suggest that smoking cessation treatments designed to reduce cue reactivity focus on each of these variables.

Neuropsychopharmacology (2008) 33, 2148-2157; doi: 10.1038/sj.npp. I301618; published online 7 November 2007
\end{abstract}

Keywords: cue reactivity; craving; nicotine dependence; fMRI; smoking; individual differences

\section{INTRODUCTION}

Exposure to cues previously associated with drug use results in physiological, behavioral, and subjective reactions, including craving (Carter and Tiffany, 1999). Among abstinent smokers, smoking-related cues can precipitate lapses (Shiffman et al, 1996) and cue reactivity is predictive of cessation outcomes (Payne et al, 2005; Waters et al, 2003). Recent neuroimaging studies have demonstrated that exposure to smoking cues results in the activation of brain networks associated with memory, attention, motivation, behavioral activation, and reward (Brody, 2006). In the present study, we sought to evaluate relations between brain responses to smoking cues and variables with the potential to modulate these responses.
*Correspondence: Dr FJ McClernon, Department of Psychiatry and Behavioral Sciences, Duke University Medical Center, 2424 Erwin Road, Box 270I, Durham, NC 27708, USA, Tel: + I 9196683987, Fax: + | 919668 5088, E-mail: mccle01 I@mc.duke.edu

Received 6 September 2007; accepted 4 October 2007

\section{NICOTINE DEPENDENCE}

Nicotine dependence is characterized by a number of factors including compulsive use of tobacco, difficulty refraining from use in situations where it is prohibited, and withdrawal following abstinence (eg upon waking in the morning) (APA, 1994; Hughes, 2006). Nicotine dependence is typically measured via self-report using measures such as the Fagerström test of nicotine dependence (FTND; Heatherton et al, 1991) and a number of studies have reported positive relations between FTND score and cue reactivity (Payne et al, 1996). However, some studies have not observed correlations between severity of nicotine dependence and cue-provoked craving (Waters et al, 2004) while others have found lighter smokers (with lower nicotine dependence scores) to exhibit greater attentional bias to smoking cues (Hogarth et al, 2003).

\section{WITHDRAWAL SYMPTOMS AND CRAVING}

Abstinence from smoking results in increases in withdrawal signs and symptoms including depressed, anxious, or irritable mood; difficulty concentrating; and disturbed 
appetite (APA, 1994). Craving for cigarettes, while not typically included in nosologies, is often considered a symptom of withdrawal (Hughes, 2007).

Little is known about how abstinence-induced withdrawal symptoms and craving modulate reactivity to drug cues. The effects of smoking abstinence on cue reactivity have been mixed, with studies showing abstinence to amplify (Alsene et al, 2003), having a trend toward amplifying (Sayette and Hufford, 1994), or having no effect (Drobes and Tiffany, 1997; Maude-Griffin and Tiffany, 1996) on the amplitude of cue-provoked craving and/or other measures of reactivity. While putatively, abstinence-induced negative affect might be expected to potentiate cue reactivity, this question has not been addressed in the literature.

\section{SEX DIFFERENCES}

The few studies that have reported sex differences in cue reactivity have typically observed greater self-report and physiological responses to cues among female subjects. In a study in which participants were exposed to smoking paraphernalia, female participants exhibited greater craving for cigarettes and increased salivation compared to male participants (Field and Duka, 2004). In another study, no sex differences in cue-provoked craving were observed, but women exhibited greater mean arterial pressure-a measure of physiological arousal - than men in response to cues (Niaura et al, 1998). These findings are consistent with research suggesting that conditioned sensory cues may play a greater role in smoking behavior in women than in men (Perkins et al, 2001).

\section{INDIVIDUAL DIFFERENCES IN BRAIN RESPONSES TO DRUG CUES}

While a number of studies have investigated smoker $v s$ nonsmoker differences in brain cue reactivity (Brody, 2006), only a handful have examined individual differences in brain reactivity among users. In one study of smokers (Smolka et al, 2006), increased brain activity in response to smoking cues was observed among more nicotine-dependent smokers in areas supporting attention (eg anterior cingulate and parietal cortex) and in regions associated with motoric functions (eg motor cortex and supplementary motor cortex). With regard to sex differences, cocainedependent men and women have been shown to exhibit different patterns of responses to cocaine cues with women exhibiting greater activation in frontal brain regions, but less activation in amygdala, insula, and ventral anterior cingulate (Kilts et al, 2004). Sex differences in brain cue reactivity have not been addressed among smokers.

\section{AIMS OF THE CURRENT ANALYSIS}

The present analysis was designed to evaluate relations between brain responses to smoking cues and nicotine dependence; self-report craving and negative affect prior to scanning; and sex. The sample consisted of adult smokers drawn from two previous imaging studies (McClernon et al, 2005b, 2007) in which the same task, scanner, and scanning parameters were used. In our analyses we examined the effects of each individual difference factor and withdrawal symptom while controlling for all other variables to evaluate the unique relation of each with brain responses. Though correlates of brain responses to sustained presentations of smoking cues have been studied (Brody et al, 2002; Smolka et al, 2006), the present analysis is unique in that transient brain responses to discrete smoking stimuli served as the dependent variable.

\section{MATERIALS AND METHODS}

\section{Participants}

Participants were pooled from two separate studies. In study 1 (McClernon et al, 2005b) smokers completed two scanning sessions-once following smoking as usual (satiated condition) and once following overnight abstinence (abstinent condition). The goal of the study was to evaluate the effects of acute abstinence on reactivity to smoking cues; smokers in study 1 did not indicate an intention to quit smoking. In study 2 (McClernon et al, 2007) smokers completed three scanning sessions: (1) at baseline, (2) following 2-4 weeks of a treatment designed to devalue the conditioned aspects of smoking, and (3) 4 weeks following quitting smoking. The goal of the study was to evaluate the effects of smoking cessation treatment and abstinence on brain responses to smoking cues. Accordingly, smokers in study 2 were recruited if they desired to quit smoking. For the analyses presented here, data were pooled from the satiated condition from study $1(n=13)$ and the baseline condition from study $2(n=18)$ since variables such as breath carbon monoxide (CO), withdrawal symptoms, and other variables were most similar during these conditions.

To participate, the following criteria were required in both studies: (1) smoking of at least 15 cigarettes per day for at least 2 years, (2) an afternoon breath $\mathrm{CO}$ level $>15$ p.p.m., (3) right-handed, (4) no serious health problems, (5) not taking medications altering CNS functioning, (6) testing negative for illicit drug use, and (7) not having any condition making MRI research unsafe. Female participants of childbearing potential were required to test negative for pregnancy at screening and in the $72 \mathrm{~h}$ prior to scanning. Potential participants were scheduled for a screening visit at which they read and signed an institutional review board-approved informed consent form, provided a urine sample that was analyzed for illicit drugs, and provided information regarding health and smoking history.

\section{Procedures}

The procedures for scanning sessions were nearly identical across the two studies. At the beginning of each fMRI session, participants completed measures of self-report withdrawal symptoms, provided breath samples, and were escorted to the scanning facility. Total time in the scanner was approximately $1.5 \mathrm{~h}$. During the satiated condition of study 1, participants were required to smoke immediately prior to entering the hospital, whereas in study 2 participants were instructed to not smoke for $2 \mathrm{~h}$ prior to scanning. 


\section{Event-Related Cue Task}

Photographic smoking, control, and target cues were presented during functional imaging using previously established methods (McClernon et al, 2005a, 2007). Smoking cues $(n=60)$ consisted of full-color pictures of smoking-related objects (eg lit cigarettes) and people smoking cigarettes. Control cues $(n=60)$ consisted of pictures of everyday objects (eg car keys, stapler) and people engaged in everyday activities (eg talking on phone). Targets $(n=15)$ were full-color pictures of animals. Participants were instructed to press a button whenever they saw a target. The inclusion of targets ensured that participants maintained attention. Stimuli included pictures from a variety of sources (Gilbert and Rabinovich, 1999; Lang et al, 1995; McClernon et al, 2005b).

All visual stimuli were projected onto a screen behind the participant's head, which the participant viewed using mirrored goggles. During each of the nine functional runs, 15 images were presented with approximately half being smoking cues, half control cues, and 1-2 targets, for a total of 135 cues per session. Each participant was presented with the same cues at each session, however, the order of presentation varied across sessions. Picture cues were presented for $4 \mathrm{~s}$ with a variable 18-22 s stimulus onset asynchrony. Total task time was $52.2 \mathrm{~min}$.

\section{Imaging Parameters}

A 1.5 T GE NVi Signa scanner with $41 \mathrm{mT} / \mathrm{m}$ gradients was used for image acquisition. Each participant's head was held in place using a vacuum-pack system to minimize head motion. Following a localizer series, blood oxygen leveldependent (BOLD) functional images were collected for 28 contiguous slices ( $4 \mathrm{~mm}$ thick) parallel to the horizontal plane connecting the anterior and posterior commissures. A spiral-out gradient-echo pulse sequence sensitive to BOLD contrast was used, with $\mathrm{TR}=2000 \mathrm{~ms}, \mathrm{TE}=40 \mathrm{~ms}$, $\mathrm{FOV}=25.6 \mathrm{~cm}$, matrix $=64 \times 64$, flip angle $=90^{\circ}$, and inplane resolution $=4 \mathrm{~mm}^{2}$. After completion of nine runs of the functional data collection, T1-weighted structural imaging was conducted on the same slices as the functional images (ie 28 slices, $4 \mathrm{~mm}$ thick) with $\mathrm{TR}=450 \mathrm{~ms}$, $\mathrm{TE}=20 \mathrm{~ms}, \quad \mathrm{FOV}=25.6 \mathrm{~cm}$, matrix $=256 \times 256$, and inplane resolution $=1 \mathrm{~mm}^{2}$.

\section{Questionnaires}

A version of the Shiffman-Jarvik Withdrawal Questionnaire (SJWQ; Shiffman and Jarvik, 1976) modified by Rose et al (1990) was used. This is a 32-item self-report measure of craving, arousal, negative affect, positive affect, habit withdrawal, and hunger. Participants completed the questionnaire at the beginning of each session. Preliminary analyses of SJWQ data observed significant correlation between negative affect and arousal $(r=0.45, p<0.01)$. Thus, arousal and other scales less central to the clinical manifestation of nicotine withdrawal were not included in the regression analysis. Nicotine dependence was measured using the six-item FTND (Heatherton et al, 1991).

\section{Data Processing and Analysis}

Functional data preprocessing was completed using statistical parametric mapping software (SPM2; Wellcome Department of Imaging Neuroscience, London) to remove noise and artifacts. The first four volumes of each run were discarded to allow for T1 stabilization. All functional images underwent correction for acquisition timing and for head motion using rigid-body rotation and translation (Friston et al, 1994). Each participant's data were then subsequently warped into a standard stereotaxic space (Montreal Neurological Institute) with an isotropic $3 \times 3 \times 3 \mathrm{~mm}$ voxel size and smoothed with an $8 \mathrm{~mm}$ Gaussian filter.

Each participant's data were then entered into a first-level voxel-by-voxel analysis using the general linear model (Friston et al, 1994) with each cue type (smoke, control, target) coded as a separate regressor. The presentation of each cue was treated as an event and modeled with a $\delta$ function convolved with a canonical hemodynamic response function. A smoking cue $>$ control cue contrast image was created for each participant and input into a random effects multiple regression analysis with four covariates (FTND, SJWQ craving, SJWQ negative affect, and sex). Correlations between each covariate and brain responses to cues were conducted after controlling for all other covariates. 'Study' was included as a nuisance variable across all analyses. Positive and negative correlations were explored for each of the covariates of interest. A gray matter mask was applied to reduce the number of comparisons and resulting activations were considered significant at a $p \leqslant 0.005$ (uncorrected) with a minimum cluster extent threshold of 10 contiguous voxels.

\section{RESULTS}

\section{Sample Characteristics}

Sample characteristics can be found in Table 1. Preliminary analyses indicated that one participant was a multivariate outlier and was thus excluded from further analyses. Thus, the final sample consisted of 30 smokers. The sample was moderately dependent, smoking approximately 24 cigarettes per day for 18 years and having a mean FTND score of 6.37. Significant differences between the two study groups were observed for age, years smoked, and Shiffman-Jarvik craving and negative affect.

\section{fMRI Results}

Tables 2-5 show brain areas where the smoking cue $>$ control cue contrast was significantly correlated (both positively and negatively) with the four covariates of interest (for results of smoking $v s$ control cue contrasts, see Supplementary Table S6 and Supplementary Figure S3). Figure 1 illustrates clusters of significant correlations between cue reactivity and each covariate of interest in seven representative brain slices.

Positive correlations between FTND scores and smoking $>$ control cue contrasts were found in right anterior cingulate gyrus (ACG; BA 32; Figure 2a), right orbitofrontal cortex (OFC; BA 11), left inferior occipital gyrus (IOG; BA $18 / 19)$, left globus pallidus (GP), and right caudate (Cd). 
Table I Participant Characteristics for the Total Sample and Study

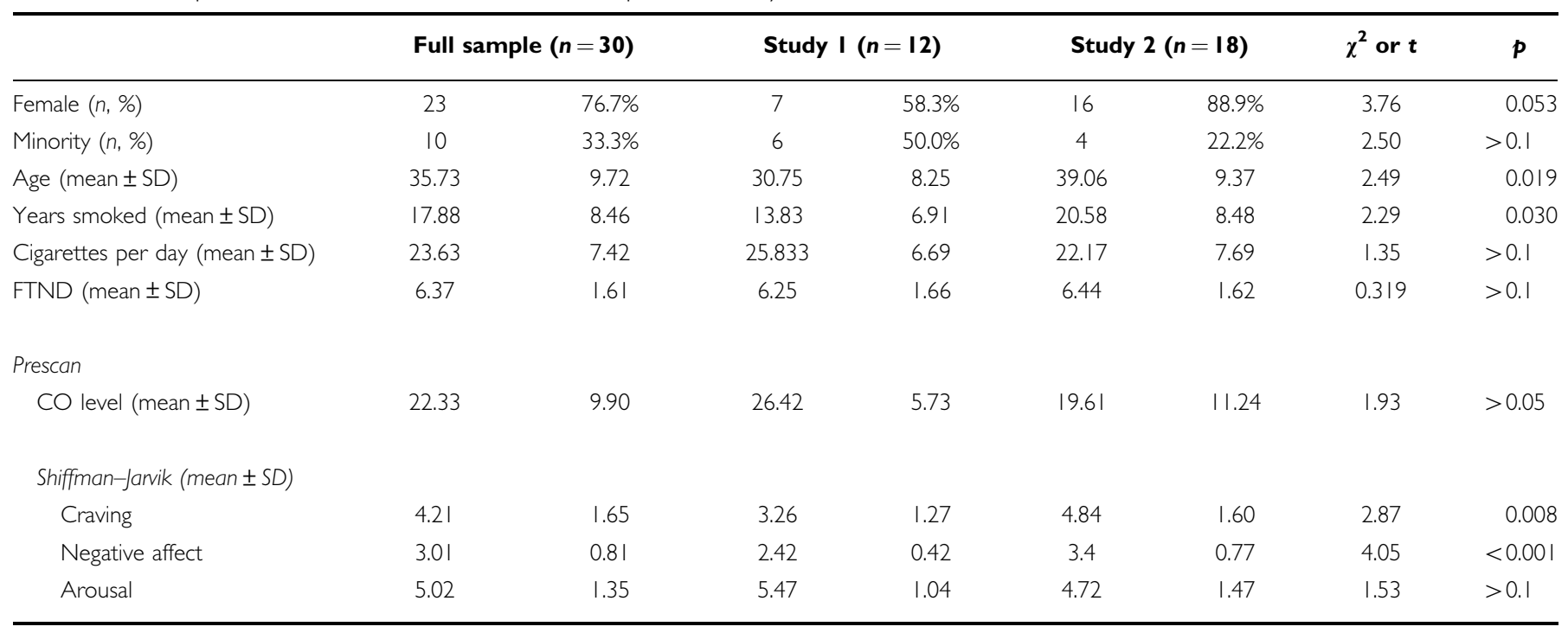

Abbreviation: FTND, Fagerström test of nicotine dependence.

$\chi^{2}$ (gender, race) and $t$-tests (age, years smoked, cigarettes per day, FTND, and prescan CO levels and Shiffman-Jarvik Withdrawal Questionnaire) were conducted to evaluate group differences.

Table 2 Brain Areas Where Cue Reactivity to Smoking Cues vs Control Cues as Measured by BOLD Signal Was Significantly Correlated with Degree of Nicotine Dependence

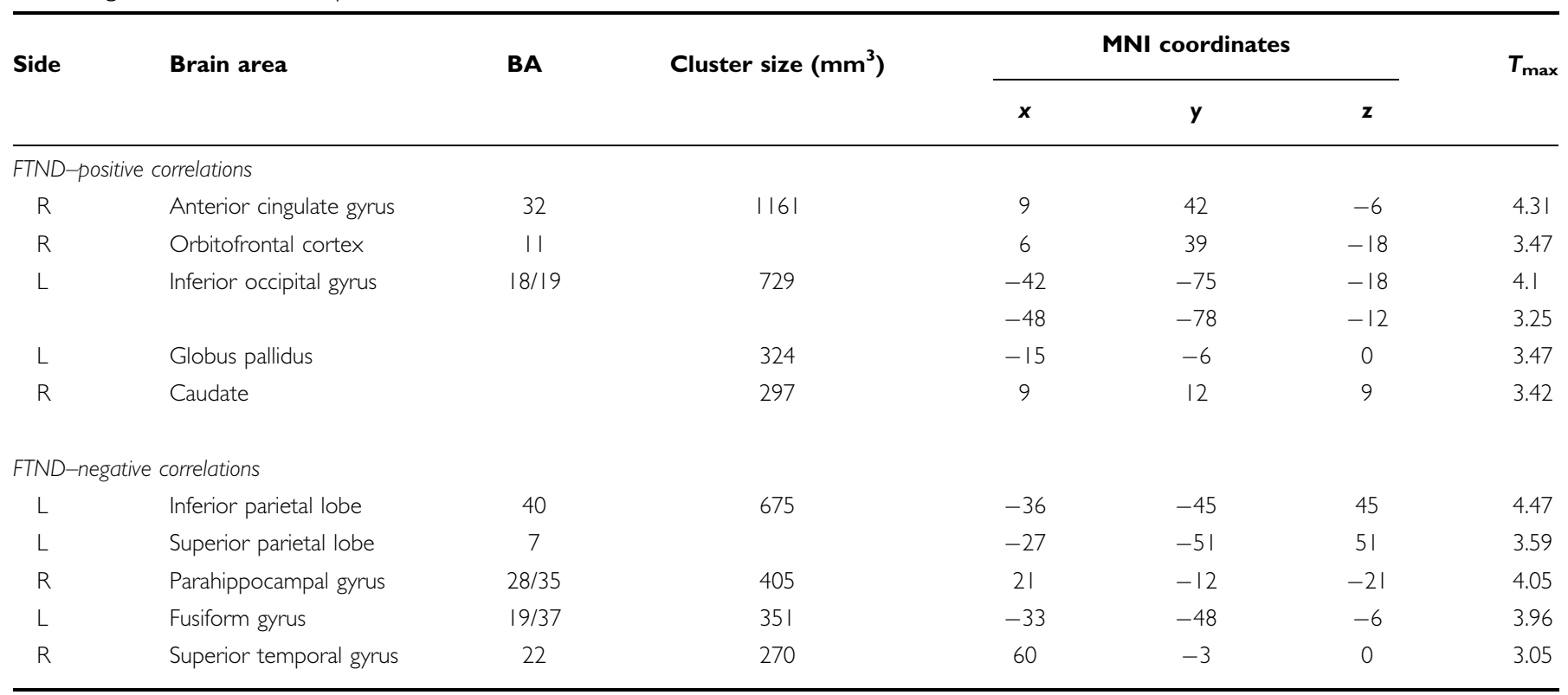

Abbreviations: MNI, Montreal Neurological Institute; BA, Brodmann Area.

$p \leqslant 0.005$ (uncorrected), minimum cluster size $\geqslant 10$ voxels.

Conversely, a negative relationship was found between nicotine dependence and cue reactivity within left inferior parietal lobe (IPL; BA 40), left superior parietal lobe (BA 7), right parahippocampal gyrus (PHG; BA 28/35), left fusiform gyrus (FG; BA 19/37), and right superior temporal gyrus (STG; BA 22).

No significant positive correlations were found between self-report craving and BOLD signal. However, significant negative correlations were observed in left ventral striatum (vSTR; Figure 2b), right medial occipital gyrus (MOG; BA 19), and in right GP.
Negative affect was found to be positively correlated with brain responses to smoking cues in left hippocampus (HC) and left OFC (BA 11). Negative correlations were found in left superior frontal gyrus (SFG; BA 6), left IPL (BA 40), and left inferior frontal gyrus (IFG; BA 47).

Differences between sexes in response to smoking cues were found in multiple regions. Women showed significantly larger cue reactivity compared to men in right putamen (Put), bilateral cuneus (Cun; BA 18/19; Figure 2c), left STG (BA 38), left middle temporal gyrus (BA 21), and 
Table 3 Brain Areas Where Cue Reactivity to Smoking Cues vs Control Cues as Measured by BOLD Signal Was Significantly Correlated with Prescan Craving Levels

\begin{tabular}{lllll}
\hline Side $\quad$ Brain area & BA & Cluster size $\left(\mathrm{mm}^{3}\right)$ & MNI coordinates & y \\
\cline { 3 - 4 } & & & $x$
\end{tabular}

Craving-positive correlations

No significant activations

\begin{tabular}{|c|c|c|c|c|c|c|c|}
\hline $\mathrm{R}$ & Medial Occipital Gyrus & 19 & 324 & 51 & -78 & 9 & 3.75 \\
\hline $\mathrm{R}$ & Globus Pallidus & & 297 & 15 & 3 & 0 & 3.7 \\
\hline
\end{tabular}

Abbreviations: MNI, Montreal Neurological Institute; BA, Brodmann Area.

$p \leqslant 0.005$ (uncorrected), minimum cluster size $\geqslant 10$ voxels.

Table 4 Brain Areas Where Cue Reactivity to Smoking Cues vs Control Cues as Measured by BOLD Signal Was Significantly Correlated with Prescan Negative Affect Reporting

\begin{tabular}{|c|c|c|c|c|c|c|c|}
\hline \multirow{2}{*}{ Side } & \multirow{2}{*}{ Brain area } & \multicolumn{2}{|c|}{$\begin{array}{l}\text { Cluster size } \\
\text { BA }\left(\mathrm{mm}^{3}\right)\end{array}$} & \multicolumn{3}{|c|}{ MNI coordinates } & \multirow{2}{*}{$T_{\max }$} \\
\hline & & & & $x$ & $y$ & $\mathbf{z}$ & \\
\hline \multicolumn{8}{|c|}{ Negative affect-positive correlations } \\
\hline L & Hippocampus & & 594 & -30 & -12 & -18 & 3.92 \\
\hline L & Orbitofrontal cortex & 11 & 324 & -12 & 33 & -18 & 3.37 \\
\hline \multicolumn{8}{|c|}{ Negative affect-negative correlations } \\
\hline \multirow[t]{2}{*}{ L } & Superior frontal gyrus & 6 & 1215 & -15 & -6 & 78 & 4.27 \\
\hline & & & & -15 & -3 & 66 & 3.49 \\
\hline L & Inferior parietal lobe & 40 & 432 & -51 & -45 & 54 & 4.25 \\
\hline L & Inferior frontal gyrus & 47 & 378 & -42 & 24 & -9 & 3.64 \\
\hline
\end{tabular}

Abbreviations: MNI, Montreal Neurological Institute; BA, Brodmann Area. $p \leqslant 0.005$ (uncorrected), minimum cluster size $\geqslant 10$ voxels.

left SFG (BA 6). In contrast, men showed greater cue reactivity in left $\mathrm{HC}$ and left MFG (BA 11).

Examination of correlations across covariates indicated substantial overlap between negative affect and sex. Specifically, brain regions positively associated with negative affect were also related to the male sex. To evaluate the degree of overlap in variance accounted for by these variables, conjunction analyses were conducted by inclusively masking negative affect with sex (threshold$p<0.005)$. These analyses confirmed that brain cue reactivity was positively associated with negative affect and with being male in HC $\left(T_{\max }=3.92 ; p<0.001\right.$; peak $\operatorname{voxel}_{x, y, z}=-30,-12$, and -18$)$ and OFC $\left(T_{\max }=3.37\right.$; $p=0.001$; peak voxel $\operatorname{voy}_{x, z}=-12,33$, and -18$)$.

\section{DISCUSSION}

In the present analyses we observed significant relations between transient brain responses to smoking cues and individual difference factors and withdrawal symptoms in a substantial sample of dependent smokers. After controlling for each of the other variables, positive relations were observed for self-report nicotine dependence, negative affect, and sex; negative relations were observed for each of these and also craving. The present findings suggest that a range of factors uniquely influence the neural processes underlying smoking cue reactivity.

\section{Nicotine Dependence}

In this analysis, self-reported nicotine dependence as measured by the FTND was positively correlated with frontal and occipital cortical regions and basal ganglia. Of particular note were correlations in VACG and OFC - both of which are regions previously implicated in drug dependence, cue reactivity, and conditioned reward (Brody et al, 2002; Cox et al, 2005; London et al, 2000; Rose et al, 2007). These findings suggest that higher levels of nicotine dependence may result in enhanced sensitivity to drug cues in these regions. Thus, they are consistent with previous reports of positive correlations between FTND scores and self-report cue reactivity (Payne et al, 1996) and analyses showing that highly dependent smokers are more sensitive to the conditioned aspects of smoking (Brauer et al, 2001). They are also consistent with previous reports of positive associations between sustained brain cue reactivity and nicotine dependence severity in cingulate gyrus (Smolka et al, 2006).

In addition to positive correlations between FTND scores and brain cue reactivity, we also observed negative correlations in regions including the FG, $\mathrm{PHG}$, and parietal and temporal cortical areas. Our findings suggest that individuals lower in nicotine dependence have activation in these regions following exposure to smoking cues, which is consistent with another study showing lighter smokers to bias attention to smoking cues compared to both heavy smokers and nonsmokers (Hogarth et al, 2003). These brain regions have been associated with a broad range of processes including face processing (FG; Puce et al, 1996), memory (PHG; Hayes et al, 2007), attention (parietal cortex; Woldorff et al, 2004), and language (temporal cortex; Bookheimer, 2002) but not reward, motivational, and/or emotional information processing. Thus, greater activation 
Table 5 Brain Areas Where Cue Reactivity to Smoking Cues vs Control Cues as Measured by BOLD Signal Significantly Differed Between Women and Men

\begin{tabular}{|c|c|c|c|c|c|c|c|}
\hline \multirow{2}{*}{ Side } & \multirow{2}{*}{ Brain area } & \multirow{2}{*}{ BA } & \multirow{2}{*}{ Cluster Size $\left(\mathrm{mm}^{3}\right)$} & \multicolumn{3}{|c|}{ MNI coordinates } & \multirow{2}{*}{$T_{\max }$} \\
\hline & & & & $x$ & $y$ & $\mathbf{z}$ & \\
\hline $\mathrm{R}$ & Putamen & & 567 & 27 & 12 & 3 & 4.99 \\
\hline $\mathrm{R}$ & Cuneus & 19 & 270 & 12 & -84 & 33 & 4.3 \\
\hline L & Cuneus & 18 & 459 & -18 & -102 & 9 & 3.95 \\
\hline L & Superior frontal gyrus & 6 & 297 & -15 & -9 & 75 & 3.64 \\
\hline \multicolumn{8}{|c|}{ Sex-male correlations } \\
\hline L & Hippocampus & & 648 & -30 & -12 & -18 & 4.11 \\
\hline
\end{tabular}

Abbreviations: MNI, Montreal Neurological Institute; BA, Brodmann Area.

$p \leqslant 0.005$ (uncorrected), minimum cluster size $\geqslant 10$ voxels.

in these regions may indicate that among less dependent smokers, smoking cues activate a network that elicits recall of smoking-related memories and guides attention to cues, but does not necessarily guide behavior or motivate use. Future studies that relate brain responses in these regions to other measures (eg attentional bias) and behaviors (eg smoking reinforcement) can help clarify these relations.

\section{Craving}

We observed significant negative associations between selfreport craving measured prior to scanning and brain responses to smoking cues in brain regions including vSTR, right MOG, and GP. The vSTR, which includes the nucleus accumbens, is sensitive to both reward magnitude (Galvan et al, 2005) and probability (Pagnoni et al, 2002) and has been shown to be active in response to drug cues (David et al, 2005; Kilts et al, 2001). At the same time, another event-related study observed decreased cue reactivity in the nucleus accumbens of smokers (Due et al, 2002). Our findings suggest that brain reactivity to cues in the vSTR may vary significantly as a function of prescan craving state and may thus explain differences observed across previous studies. Moreover, if vSTR activation to a cue is an index of the potential magnitude of the reward value represented by the cue, the negative correlations observed in the present study may indicate that the reward salience of smoking cues is attenuated, or even becomes negative, among smokers who are experiencing more severe prescan craving. If vSTR activation in response to a cue is an index of the mismatch between a cue and a predicted outcome (ie an index of prediction error), the present results suggest greater prediction error among smokers lower in craving. In other words, when craving is low, conditioned drug cues may represent a greater mismatch with the current drug state and thus elicit greater striatal activation. When craving is high, the presence of smoking cues is highly consistent with the current craving state and may not represent a mismatch between craving state and stimulus. Future studies that specifically manipulate craving states and the reward value of smoking cues can help dissociate reward magnitude $v \mathrm{~s}$ prediction signal in the striatum.

Unlike other variables in this analysis, no positive correlations between craving and brain responses were observed. Moreover, other studies have observed significant positive correlations between craving and indices of cue reactivity (Brody et al, 2002; Smolka et al, 2006). Several factors might account for the lack of positive associations in the current study. One possibility is that after controlling for other variables potentially related to the degree of prescanning craving such as nicotine dependence and negative affect, craving did not account for significant variability. Perhaps even more importantly, the current study evaluated the influence of prescan craving as opposed to cue-provoked craving. Thus, our lack of positive associations may simply indicate that craving measured prior to exposure to cues has a negative relationship with brain cue reactivity whereas relations between self-reports of craving provoked by cues and brain cue reactivity are more robust and positive.

\section{Negative Affect and Sex}

In this analysis, men or individuals reporting greater negative affect exhibited greater activation to cues in both $\mathrm{HC}$ and OFC. Negative affect is a central component of nicotine withdrawal (Hughes, 2007) and individuals with chronic negative affect such as individuals with major depressive disorder smoke at higher rates (Lasser et al, 2000). Regardless of the source of negative affect - either withdrawal induced or mood state - the present findings suggest that prescan negative affect may be associated with increased brain activation in response to cues. As noted above, the OFC has been implicated in appetitive behaviors including drug taking (London et al, 2000). In addition to the OFC, negative affect was associated with activation in 


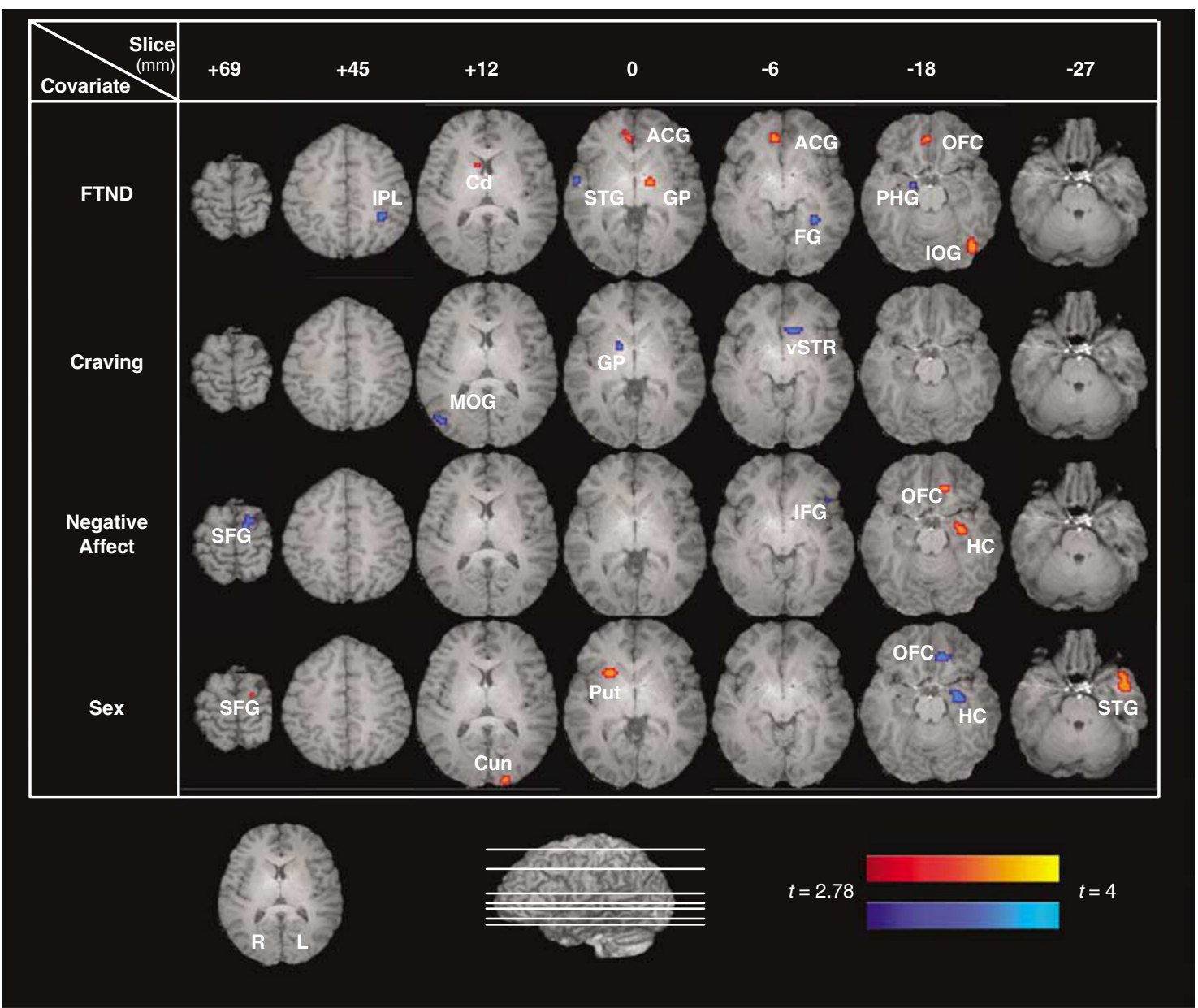

Figure I Areas of activation where cue reactivity was significantly correlated with covariates of interest. Activations in red represent where cue reactivity was positively correlated whereas areas in blue represent negative correlations. Fagerström test of nicotine dependence (FTND) was positively correlated with cue reactivity in right caudate (Cd), right anterior cingulate gyrus (ACG; BA 32), left globus pallidus (GP), right orbitofrontal cortex (OFC; BA I I), and left inferior occipital gyrus (IOG; BA 18/19); and negatively correlated in left inferior parietal lobe (IPL; BA 40), right superior temporal gyrus (STG; BA 22), left fusiform gyrus (FG; BA 19/37), and right parahippocampal gyrus (PHG; BA 28/35). Craving was negatively correlated with cue reactivity in right medial occipital gyrus (MOG; BA 19), right GP, and left ventral striatum (VSTR). Negative affect was positively correlated with cue reactivity in left OFC (BA I I) and left hippocampus (HC); and negatively correlated in left superior frontal gyrus (SFG; BA 6), inferior frontal gyrus (IFG; BA 47). Female sex (red) was positively correlated with cue reactivity in left SFG (BA 6), left cuneus (Cun; BA 18), right putamen (Put), and left STG (BA 38); male sex (blue) was positively correlated in left OFC (BA II) and left HC.

the HC-a region central to memory functions. Together, these findings suggest that greater levels of negative affect may be associated with increases in appetitive motivation provoked by smoking cues and memorial processes that support drug use.

In addition to self-report negative affect, being male was also associated with greater activation in OFC and HC. This overlap in findings for negative affect and sex is interesting given that each of these factors was controlled for in the analysis of the other. These two variables were only modestly correlated with one another $(r=0.403)$ with women reporting higher levels of negative affect $($ mean $=3.18 ; \quad \mathrm{SD}=0.81) \quad$ than men $\quad($ mean $=2.43$; $\mathrm{SD}=0.47)$ prior to scanning. Further, if collinearity had been high, these two variables would not account for unique variance in brain activation in these two regions since their effects would cancel one another out. Thus, these findings suggest that reactivity to smoking cues in these regions may be modulated to some degree by both negative affect and sex variables. Additional research and analyses including both of these factors will likely be necessary to clarify these findings.

Female sex was associated with cue reactivity in specific brain regions that did not overlap with other findings. For instance, female sex was associated with activation in Cun, STG, and Put. The Cun, which subserves visuospatial attention, has been shown to be active in response to smoking cues in several previous studies of smoking cue reactivity (Brody et al, 2007; Smolka et al, 2006; Wilson et al, 2005). The function of the STG in smoking cue reactivity is unclear. This region is associated with speech processing and greater regional cerebral blood flow in response to cocaine cues was previously observed in a female sample (Kilts et al, 2004). However, in that study, cues were presented via audiotaped scripts. Finally, the Put - which makes up part of the dorsal striatum - has 

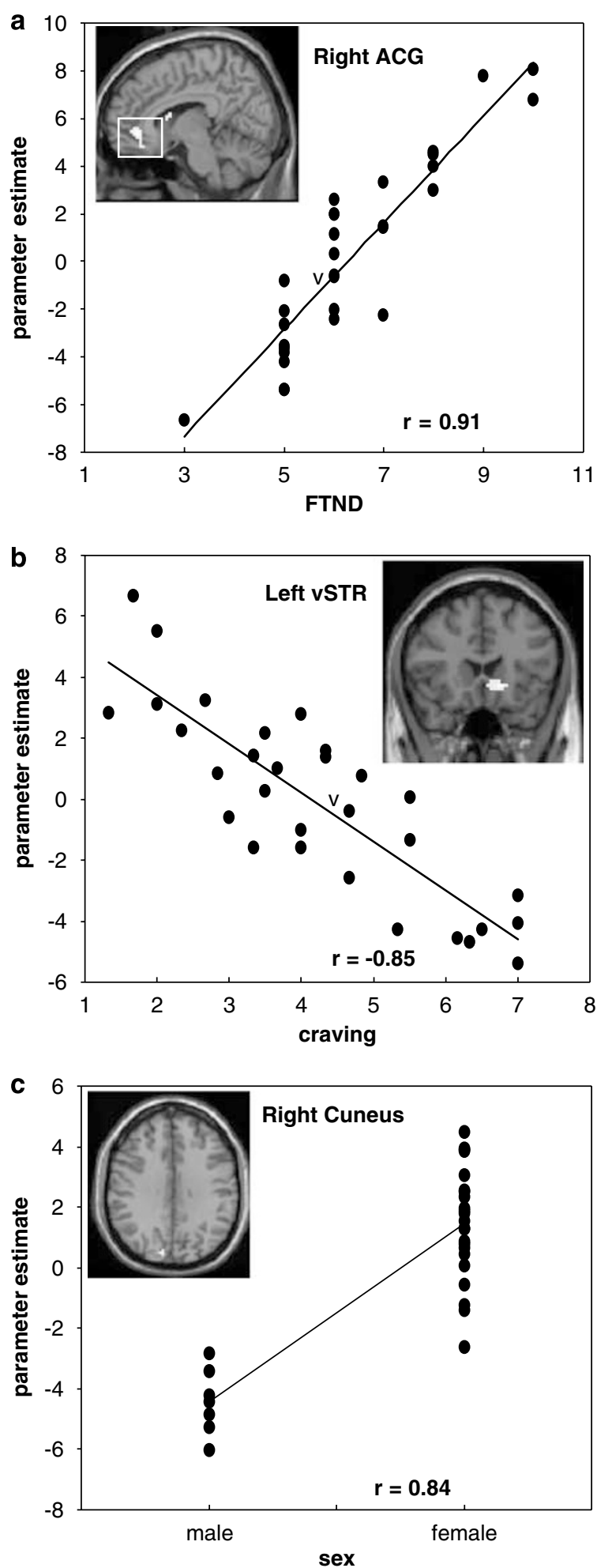

Figure 2 Graphical representations of the correlations observed between BOLD activation to smoking > control cue contrasts and (a) Fagerström test of nicotine dependence (FTND) scores in right anterior cingulate gyrus (ACG; BA 32), (b) self-report measures of prescan craving (Shiffman-Jarvik Withdrawal Questionnaire) in left ventral striatum (VSTR), and (c) sex in right cuneus (BA I8).

been implicated in craving for drugs including alcohol and cocaine (Heinz et al, 2005; Volkow et al, 2006; Wong et al, 2006).
When considered together with the findings for men, the present study suggests that sex differences in reactivity to cues are likely modulated via distinct neural circuits but may interact in complex ways with other individual difference variables including negative affect. Very few studies have observed sex differences in cue-provoked craving (Field and Duka, 2004) or other forms of cue reactivity (Niaura et al, 1998). However, given the observation of sex differences in the present study, it could be hypothesized that men and women engage different neural circuits in the processing of cues that lead ultimately to similar levels of subjective craving. The lack of a measure of cue-provoked craving in the present study precluded us from testing this hypothesis. Future imaging studies with larger samples that include measures of cue-provoked craving can help clarify potential neural markers of sex differences in cue reactivity.

\section{LIMITATIONS}

The present analysis has a number of strengths, including the substantial sample size for an fMRI study, use of multivariate regression to isolate unique relations, and examination of sex differences. However, it is also limited by a number of factors. First, the present analysis aggregated data from two different studies. There were some significant differences between the two samples (eg age) but there were also likely unmeasured differences (eg motivation to quit). While potentially problematic, as in previous aggregate analyses (Garavan et al, 2006), the inclusion of study as a nuisance variable should have accounted for variability due to these group differences. Future studies that evaluate how individual differences in motivation to quit, age, and other factors influence brain cue reactivity are warranted. Second, the relatively smaller number of men in the study ( $n=8 v s n=22$ women) means that sex effects, even significant ones, should be considered tentative and require replication in larger samples. Third, while there was a degree of variability in the sample in terms of smoking history and level of nicotine dependence, the sample of smokers was moderately nicotine-dependent and thus not representative of all smokers. Finally, to avoid type II error, we extended our analyses to all gray matter areas of the brain. While our chosen $\alpha$ level $(p<0.005)$ is consistent with similar analyses of brain cue reactivity (Smolka et al, 2006) and provided adequate protection from type I error, future studies could limit analyses to a priori regions identified in the present study.

\section{CONCLUSIONS}

The present analyses sought to identify unique associations between brain responses to smoking cues and potentially important factors including nicotine dependence, withdrawal symptoms, and sex. A number of interesting relations emerged between these factors and activation in limbic and reward regions including ventral ACG, vSTR, and OFC. These findings suggest that biological (eg sex), smoking history-related (eg nicotine dependence), and withdrawal symptoms (eg craving, negative affect) can have unique influences on brain cue reactivity. Likewise, the 
influence of each of these factors on brain cue reactivity may represent a distinct endophenotype, each of which is under the control of a distinct set of genetic and environmental influences. Future research can evaluate this question more fully and can also evaluate the effects of behavioral and pharmacological interventions on relations between brain cue reactivity and individual difference factors and withdrawal symptoms.

\section{ACKNOWLEDGEMENTS}

We thank Mr Berry Hiott for collection of fMRI data. This research was supported by NIDA grants R03DA016212 and K23DA017261 (to Dr McClernon) and by an unrestricted donation from Vector Tobacco Co. (to Dr Rose).

\section{DISCLOSURE/CONFLICT OF INTEREST}

Dr McClernon reports having research funding from the National Institute on Drug Abuse, the National Alliance for Research on Schizophrenia and Depression, and the Atkins Foundation; and receiving consulting fees from Ely Lilly and Company. Dr Rose reports research funding from Vector Tobacco Co., and Philip Morris Inc.; and receiving consulting fees from Novartis Pharmaceuticals, GlaxoSmithKline Inc., Medacorp, Pharmalink, and Noble Medical Consulting Group. Ms Kozink reports no conflicts of interest.

\section{REFERENCES}

Alsene KM, Li Y, Chaverneff F, de Wit H (2003). Role of abstinence and visual cues on food and smoking craving. Behav Pharmacol 14: $145-151$.

APA (1994). Diagnostic and Statistical Manual of Mental Disorders, 4th edn. American Psychiatric Association: Washington, DC.

Bookheimer S (2002). Functional MRI of language: new approaches to understanding the cortical organization of semantic processing. Annu Rev Neurosci 25: 151-188.

Brauer LH, Behm FM, Lane JD, Westman EC, Perkins C, Rose JE (2001). Individual differences in smoking reward from denicotinized cigarettes. Nicotine Tob Res 3: 101-109.

Brody AL (2006). Functional brain imaging of tobacco use and dependence. J Psychiatr Res 40: 404-418.

Brody AL, Mandelkern MA, London ED, Childress AR, Lee GS, Bota RG et al (2002). Brain metabolic changes during cigarette craving. Arch Gen Psychiatry 59: 1162-1172.

Brody AL, Mandelkern MA, Olmstead RE, Jou J, Tiongson E, Allen V et al (2007). Neural substrates of resisting craving during cigarette cue exposure. Biol Psychiatry 62: 642-651.

Carter BL, Tiffany ST (1999). Meta-analysis of cue-reactivity in addiction research. Addiction 94: 327-340.

Cox SM, Andrade A, Johnsrude IS (2005). Learning to like: a role for human orbitofrontal cortex in conditioned reward. J Neurosci 25: 2733-2740.

David SP, Munafo MR, Johansen-Berg H, Smith SM, Rogers RD, Matthews PM et al (2005). Ventral striatum/nucleus accumbens activation to smoking-related pictorial cues in smokers and nonsmokers: a functional magnetic resonance imaging study. Biol Psychiatry 58: 488-494.

Drobes DJ, Tiffany ST (1997). Induction of smoking urge through imaginal and in vivo procedures: physiological and self-report manifestations. J Abnorm Psychol 106: 15-25.
Due DL, Huettel SA, Hall WG, Rubin DC (2002). Activation in mesolimbic and visuospatial neural circuits elicited by smoking cues: evidence from functional magnetic resonance imaging. Am J Psychiatry 159: 954-960.

Field M, Duka T (2004). Cue reactivity in smokers: the effects of perceived cigarette availability and gender. Pharmacol Biochem Behav 78: 647-652.

Friston KJ, Jezzard P, Turner R (1994). Analysis of functional MRI time-series. Hum Brain Mapp 1: 153-171.

Galvan A, Hare TA, Davidson M, Spicer J, Glover G, Casey BJ (2005). The role of ventral frontostriatal circuitry in rewardbased learning in humans. J Neurosci 25: 8650-8656.

Garavan H, Hester R, Murphy K, Fassbender C, Kelly C (2006). Individual differences in the functional neuroanatomy of inhibitory control. Brain Res 1105: 130-142.

Gilbert DG, Rabinovich NE (1999). International Smoking Image Series, Version 1.2, 1.2 edn. Integrative Neuroscience Laboratory, Southern Illinois University-Carbondale: Carbondale, IL.

Hayes SM, Nadel L, Ryan L (2007). The effect of scene context on episodic object recognition: parahippocampal cortex mediates memory encoding and retrieval success. Hippocampus 17: 873889.

Heatherton TF, Kozlowski LT, Frecker RC, Fagerstrom KO (1991). The fagerstrom test for nicotine dependence: a revision of the fagerstrom tolerance questionnaire. $\mathrm{Br}$ J Addict 86: 1119-1127.

Heinz A, Siessmeier T, Wrase J, Buchholz HG, Grunder G, Kumakura Y et al (2005). Correlation of alcohol craving with striatal dopamine synthesis capacity and $\mathrm{D} 2 / 3$ receptor availability: a combined [18F]DOPA and [18F]DMFP PET study in detoxified alcoholic patients. Am J Psychiatry 162: 1515-1520.

Hogarth LC, Mogg K, Bradley BP, Duka T, Dickinson A (2003). Attentional orienting towards smoking-related stimuli. Behav Pharmacol 14: 153-160.

Hughes JR (2006). Should criteria for drug dependence differ across drugs? Addiction 101(Suppl 1): 134-141.

Hughes JR (2007). Effects of abstinence from tobacco: valid symptoms and time course. Nicotine Tob Res 9: 315-327.

Kilts CD, Gross RE, Ely TD, Drexler KP (2004). The neural correlates of cue-induced craving in cocaine-dependent women. Am J Psychiatry 161: 233-241.

Kilts CD, Schweitzer JB, Quinn CK, Gross RE, Faber TL, Muhammad F et al (2001). Neural activity related to drug craving in cocaine addiction. Arch Gen Psychiatry 58: 334-341.

Lang PJ, Bradley MM, Cuthbert BN (1995). International Affective Picture System (IAPS): Technical Manual and Affective Ratings. NIMH Center for the Study of Emotion and Attention, University of FL: Gainesville, FL.

Lasser K, Boyd JW, Woolhandler S, Himmelstein DU, McCormick D, Bor DH (2000). Smoking and mental illness: a populationbased prevalence study. JAMA 284: 2606-2610.

London ED, Ernst M, Grant S, Bonson K, Weinstein A (2000). Orbitofrontal cortex and human drug abuse: functional imaging. Cereb Cortex 10: 334-342.

Maude-Griffin P, Tiffany ST (1996). Production of smoking urges through imagery: the impact of affect and smoking abstinence. Exp Clin Psychopharmacol 4: 198-208.

McClernon FJ, Beckham JC, Mozley SL, Feldman ME, Vrana SR, Rose JE (2005a). The effects of trauma recall on smoking topography in posttraumatic stress disorder and non-posttraumatic stress disorder trauma survivors. Addict Behav 30: 247-257.

McClernon FJ, Hiott FB, Huettel SA, Rose JE (2005b). Abstinenceinduced changes in self-report craving correlate with eventrelated FMRI responses to smoking cues. Neuropsychopharmacology 30: 1940-1947.

McClernon FJ, Hiott FB, Liu J, Salley AN, Behm FM, Rose JE (2007). Selectively reduced responses to smoking cues in amygdala following extinction-based smoking cessation: results of a preliminary fMRI study. 12: 503-512. 
Niaura R, Shadel WG, Abrams DB, Monti PM, Rohsenow DJ, Sirota A (1998). Individual differences in cue reactivity among smokers trying to quit: effects of gender and cue type. Addict Behav 23: 209-224.

Pagnoni G, Zink CF, Montague PR, Berns GS (2002). Activity in human ventral striatum locked to errors of reward prediction. Nat Neurosci 5: 97-98.

Payne TJ, Smith PO, Adams SG, Diefenbach L (2005). Pretreatment cue reactivity predicts end-of-treatment smoking. Addict Behav 31: 702-710.

Payne TJ, Smith PO, Sturges LV, Holleran SA (1996). Reactivity to smoking cues: mediating roles of nicotine dependence and duration of deprivation. Addict Behav 21: 139-154.

Perkins KA, Gerlach D, Vender J, Grobe J, Meeker J, Hutchison S (2001). Sex differences in the subjective and reinforcing effects of visual and olfactory cigarette smoke stimuli. Nicotine Tob Res 3: 141-150.

Puce A, Allison T, Asgari M, Gore JC, McCarthy G (1996). Differential sensitivity of human visual cortex to faces, letterstrings, and textures: a functional magnetic resonance imaging study. J Neurosci 16: 5205-5215.

Rose JE, Behm FM, Salley AN, Bates JE, Coleman RE, Hawk TC. et al (2007). Regional brain activity correlates of nicotine dependence. Neuropsychopharmacology (in press).

Rose JE, Levin ED, Behm FM, Adivi C, Schur C (1990). Transdermal nicotine facilitates smoking cessation. Clin Pharmacol Ther 47: 323-330.

Sayette MA, Hufford MR (1994). Effects of cue exposure and deprivation on cognitive resources in smokers. J Abnorm Psychol 103: $812-818$.
Shiffman S, Paty JA, Gnys M, Kassel JA, Hickcox M (1996). First lapses to smoking: within-subjects analysis of real-time reports. J Consult Clin Psychol 64: 366-379.

Shiffman SM, Jarvik ME (1976). Smoking withdrawal symptoms in two weeks of abstinence. Psychopharmacology (Berl) 50: 35-39.

Smolka MN, Buhler M, Klein S, Zimmermann U, Mann K, Heinz A et al (2006). Severity of nicotine dependence modulates cueinduced brain activity in regions involved in motor preparation and imagery. Psychopharmacology (Berl) 184: 577-588.

Volkow ND, Wang GJ, Telang F, Fowler JS, Logan J, Childress AR et al (2006). Cocaine cues and dopamine in dorsal striatum: mechanism of craving in cocaine addiction. J Neurosci 26: 65836588.

Waters AJ, Shiffman S, Sayette MA, Paty JA, Gwaltney CJ, Balabanis MH (2003). Attentional bias predicts outcome in smoking cessation. Health Psychol 22: 378-387.

Waters AJ, Shiffman S, Sayette MA, Paty JA, Gwaltney CJ, Balabanis MH (2004). Cue-provoked craving and nicotine replacement therapy in smoking cessation. J Consult Clin Psychol 72: 1136-1143.

Wilson SJ, Sayette MA, Delgado MR, Fiez JA (2005). Instructed smoking expectancy modulates cue-elicited neural activity: a preliminary study. Nicotine Tob Res 7: 637-645.

Woldorff MG, Hazlett CJ, Fichtenholtz HM, Weissman DH, Dale AM, Song AW (2004). Functional parcellation of attentional control regions of the brain. J Cogn Neurosci 16: 149-165.

Wong DF, Kuwabara H, Schretlen DJ, Bonson KR, Zhou Y, Nandi A et al (2006). Increased occupancy of dopamine receptors in human striatum during cue-elicited cocaine craving. Neuropsychopharmacology 12: 2716-2727.

Supplementary Information accompanies the paper on the Neuropsychopharmacology website (http://www.nature.com/npp) 